\title{
PREVALENCE AND CHARACTERISTICS OF METHICILLIN-RESISTANT STAPHYLOCOCCI IN GOATS ON THE ISLAND OF TENERIFE, SPAIN
}

\author{
Rossana ABREU ${ }^{1,2}$, Cristobalina RodRÍGUEZ-ÁLVAREZ ${ }^{1}$, María LECUONA ${ }^{2}$, \\ Beatriz CASTRO-HERNÁNDEZ ${ }^{2}$, Juan Carlos GONZÁLEZZ ${ }^{3}$, Armando AGUIRRE-JAIME ${ }^{4}$ and \\ Ángeles ARIAS ${ }^{1 *}$ \\ ${ }^{1}$ Department of Preventive Medicine and Public Health, University of La Laguna, \\ Campus de Ofra S/N, Santa Cruz de Tenerife 38071, Spain; ${ }^{2}$ University Hospital of \\ the Canary Islands, San Cristobal de La Laguna, Tenerife, Canary Islands, Spain; \\ ${ }^{3}$ Canary Islands Health Service, Santa Cruz de Tenerife, Canary Islands, Spain; \\ ${ }^{4}$ Institute of Care Research, Nurses Association of Santa Cruz de Tenerife, Spain
}

(Received 12 March 2019; accepted 2 May 2019)

The aim of this study was to determine the prevalence of methicillinresistant Staphylococcus (MRS) in healthy goats on the Island of Tenerife, Spain, as well as to identify the phenotypic and genotypic characteristics of the strains found. A cross-sectional prevalence study was conducted. A total of 158 goats from 15 different farms were sampled between September 2017 and January 2018. The percentage of positive samples of methicillin-resistant Staphylococcus aureus (MRSA) was 15.8\% (25/158) and that of methicillin-resistant coagulasenegative staphylococci (MRCoNS) was 6.9\% (11/158). All MRSA isolates from goats belonged to one clonal group showing Multi-Locus Sequence type 398. All strains studied $(\mathrm{n}=36)$ were resistant to non-carbapenem beta-lactam antibiotics and susceptible to teicoplanin, linezolid, vancomycin, rifampicin, quinupristindalfospristin and mupirocine. In MRSA isolates, the highest percentage of resistance obtained, besides beta-lactam non-carbapenem antibiotics, was to trimethoprim-sulphamethoxazole and, in the case of MRCoNS isolates, to phosphomycin and erythromycin. A total of 12 resistance patterns were obtained, presenting differences between patterns obtained for MRSA and MRCoNS, with 7 different patterns for MRSA and 5 for MRCoNS. We therefore consider it essential to expand the epidemiological study of these strains of animal origin, as well as to increase surveillance and control measures at all stages of the food chain.

Key words: Methicillin-resistant Staphylococcus aureus, methicillin-resistant coagulase-negative staphylococci, phenotypic and genotypic characteristics, goat

Methicillin resistance in Staphylococcus aureus is a serious concern in both human and veterinary medicine (Hernández-Porto et al., 2014; Goerge et al., 2017; Ortwine and Bhavan, 2018). The main gene linked to resistance to the-

“Corresponding author; E-mail: angarias@ull.edu.es; Phone: 0034 (922) 319-369 
se antibiotics is the mecA gene, found in Staphylococcal Cassette Chromosome mec (SCCmec), which are mobile genetic elements responsible for the transfer of resistance genes (Shore and Coleman, 2013) and are also part of the genome of coagulase-negative Staphylococcus (MRCoNS) species (Vanderhaeghen et al., 2014; Fernandes Dos Santos et al., 2016). Several studies indicate that, like MRSA, MRCoNS have animal production facilities as a reservoir and can be found in meat products and other foods such as the milk of ruminants (Vanderhaeghen et al., 2014; Landeta et al., 2013; Nemeghaire et al., 2014).

The presence of Staphylococcus strains, such as MRSA and MRCoNS, is widely described in mastitis of various ruminants including goats, and, as a consequence, in their milk (Chu et al., 2012; Cortimiglia et al., 2015; Mahato et al., 2017; Obaidat et al., 2018). In recent years, a great number of studies have been carried out on colonisation prevalence of multiresistant Staphylococcus strains in different farm and domestic animals in farm environments and in people in contact with them (Habrun et al., 2011; Morcillo et al., 2012; Pletinckx et al., 2013; Sun et al., 2017; Pomba et al., 2017; Rodrigues et al., 2018; Sato et al., 2018; Feld et al., 2018), mostly with reference to MRSA.

To date, however, very few studies have been conducted on the colonisation of multiresistant bacteria in goats (Aquino et al., 2012; Loncaric et al., 2013). Goat production is widespread in the Canary Islands, as it easily adapts to the weather conditions of the islands. The goat production systems are semi-intensive, where the animals graze freely and are kept in stables for the night. As the island of Tenerife is geographically remote from the European and African continents, animals are more isolated than those on the continent.

The objective of this study was to determine the prevalence of methicillinresistant Staphylococcus in healthy goats on the island of Tenerife, Spain, as well as to identify the phenotypic and genotypic characteristics of the isolated strains.

\section{Materials and methods}

\section{Collection of samples}

A cross-sectional prevalence study was conducted. A total of 158 goats were screened, representing a randomised selection of animals from 15 wean-tofinish farms for local consumption. Goat samples from farms all over the island were collected between September 2017 and January 2018. The study was authorised by the General Public Health Direction of the Health Service of Canary Islands. The sampling was done by a veterinarian of that Public Health Direction. All samples were collected with cotton-tipped swabs that were placed in Amies Rayon (deltalab ${ }^{\mathrm{TM}}$ ), stored at $4{ }^{\circ} \mathrm{C}$ and transported directly to the laboratory. 


\section{Isolation and identification of MRS isolates}

Nasal swabs were incubated in Brain-Heart Infusion (BHI) with $7 \% \mathrm{NaCl}$ for $18-24 \mathrm{~h}$ at $37^{\circ} \mathrm{C}$. Then, $10 \mu \mathrm{l}$ of the infusion was plated onto MRSA-ID culture plates (bioMérieux ${ }^{\mathrm{TM}}$ ). The plates were then incubated aerobically at $37^{\circ} \mathrm{C}$ for 24-48 h. Staphylococcus suspicious colonies were identified by morphology and growth colour. Catalase test and coagulase test agglutination (Slidex ${ }^{\mathrm{TM}} \mathrm{Staph}$ Plus, bioMérieux ${ }^{\mathrm{TM}}$ ) were performed on malachite green and white coloured colonies. Species identifications were confirmed by the Vitek II Automated Microbiology System with ID card GP (bioMerieux ${ }^{\mathrm{TM}}$ ). Methicillin resistance was confirmed by testing for the presence of penicillin-binding protein A (PBP2a) (MRSA-screen; Denka Seiken $\mathrm{Co}^{\mathrm{TM}}$, Japan) and the presence of the mecA gene was detected by Real-Time PCR (RT-PCR) (iQ ${ }^{\mathrm{TM}} 5$, BioRad). Staphylococcus aureus ATCC 29213 was used as reference strain.

\section{Molecular typing of MRSA}

DNA macro-restriction analysis by pulsed-field gel electrophoresis (PFGE). Isolation of chromosomal DNA was performed as described by Smith et al. (1988). The enzyme used for the macro-restriction was ApaI (Promega) with the following electrophoresis conditions: block I: $6 \mathrm{v} / \mathrm{cm}, 12 \mathrm{~h}, 5-15 \mathrm{sec}$; block II: $6 \mathrm{v} /$ $\mathrm{cm}, 12 \mathrm{~h}, 15-40 \mathrm{sec}$. The results were interpreted according to the criteria described by Tenover et al. (1995).

Sequencing. All MRSA isolates were analysed by multilocus sequence typing (MLST) as described previously (Enright et al., 2000). MLST was based on a sequence analysis of the internal fragments of seven housekeeping genes: $\operatorname{arcC}$ (Carbamate kinase), aroE (Shikimate dehydrogenase), glpF (Glycerol kinase), gmk (Guanylate kinase), pta (Phosphate acetyltransferase), tpi (Triosephosphate isomerase), yqi (Acetyl-coenzyme A acetyltransferase). The allelic profiles and sequence types were assigned according to the $S$. aureus MLST database (https://pubmlst.org/).

\section{Antimicrobial susceptibility testing}

Antimicrobial susceptibility was determined by the broth microdilution method using Vitek-2 AST-626 cards (bioMérieux ${ }^{\circledR}$, France). Staphylococcus aureus ATCC 29213 was used as reference strain. Strains were tested for susceptibility to beta-lactams: benzylpenicillin (PGL/PG), oxacillin (OXA); aminoglycosides: gentamicin (GM), tobramycin (TM); glycopeptides: teicoplanin (TP), vancomycin (VA); quinolone: levofloxacin (LV); lincosamide: clindamycin (CC); macrolide: erythromycin (E); rifamycin: rifampicin (RI); bacteriostatics: trimethoprim-sulphamethoxazole (SXT), fusidic acid (FA); streptogramins: quinupristin-dalfopristin (QDA); oxazolidinone: linezolid (LZ); glycylcycline: tigecycline (TGC); phosphate: phosphomycin (FM); monocarboxylic acid: mupirocin (MU). 
The breakpoints used were the same as those established by the Clinical and Laboratory Standards Institute Guidelines (2011).

\section{Statistical analysis}

The characteristics of the samples are described with the relative frequency of the categories that make up nominal variables. Frequency comparisons were performed with Fisher's Exact Test at a significance level $\mathrm{P} \leq 0.05$. Calculations were performed with the IBM $\mathrm{Co}^{\mathrm{TM}}$ statistical processing package for PC in operating environment Windows SPSS 21.0.

\section{Results}

The prevalence of methicillin-resistant Staphylococcus was 22.8\% (36/158), of which $15.8 \%(25 / 158)$ corresponded to MRSA and 6.9\% (11/158) to MRCoNS. These latter strains were found through chromogenic medium while detecting MRSA, all being positive for the mecA gene. The following species were identified: Staphylococcus lentus $(\mathrm{n}=3,27.2 \%)$, Staphylococcus sciuri $(\mathrm{n}=3,27.2 \%)$, Staphylococcus haemolyticus $(\mathrm{n}=2,18.2 \%)$, Staphylococcus auricularis $(\mathrm{n}=1$, 9.1\%), Staphylococcus gallinarum $(\mathrm{n}=1,9.1 \%)$, Staphylococcus pasteuri $(\mathrm{n}=1$, $9.1 \%)$.

Genotypic study of the MRSA strains demonstrated, according to the analysis of the patterns obtained by PFGE, that the isolates were closely related and almost all belonged to the same clone (Fig. 1). MLST confirmed that all isolates belonged to ST398 (CC398).

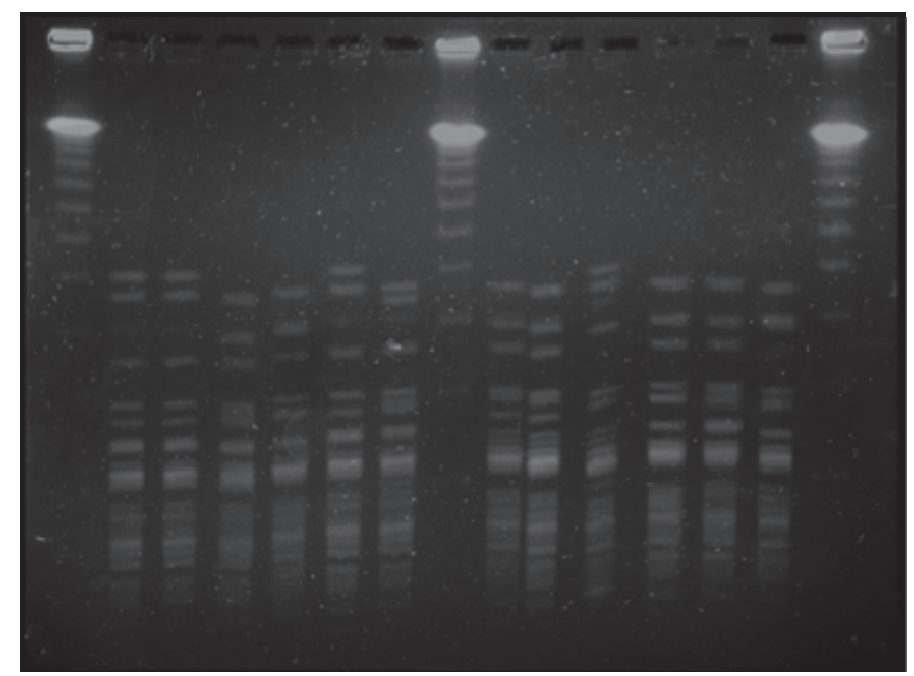

Fig. 1. Molecular typing by PFGE of ApaI-digested DNA from MRSA isolates from goats 
All strains studied $(\mathrm{n}=36)$ were resistant to beta-lactam non-carbapenem antibiotics and sensitive to teicoplanin, linezolid, vancomycin, rifampicin, quinupristin-dalfopristin, and mupirocine.

Table 1 shows the antibiotic resistance percentages of the strains tested and their distribution for MRSA and MRCoNS. Besides beta-lactam noncarbapenem antibiotics, the MRSA isolates showed the highest percentage of resistance to trimethoprim-sulphamethoxazole and the MRCoNS isolates to phosphomycin and erythromycin. There were significant differences in resistance to erythromycin $(\mathrm{P}=0.006)$, fusidic acid $(\mathrm{P}=0.023)$, phosphomycin $(\mathrm{P}=0.000)$, and trimethoprim-sulphamethoxazole $(\mathrm{P}=0.047)$.

Table 1

Antimicrobial resistance of mecA Staphylococcus isolates from goats in Tenerife, Spain

\begin{tabular}{lcccc}
\hline Antimicrobial & Total & MRSA & MRCoNS & Significance \\
& $\mathrm{n}(\%)$ & $\mathrm{n}(\%)$ & $(\mathrm{P})$ & - \\
\hline Benzylpenicillin & $36(100)$ & $25(100)$ & $11(100)$ & - \\
Oxacillin & $36(100)$ & $25(100)$ & $11(100)$ & 0.290 \\
Gentamicin & $4(10.8)$ & $4(16.0)$ & $0(0)$ & 0.290 \\
Tobramycin & $4(10.8)$ & $4(16.0)$ & $0(0)$ & 0.409 \\
Clindamycin & $9(24.3)$ & $5(20.0)$ & $4(36.4)$ & 0.006 \\
Erythromycin & $13(35.1)$ & $5(20.0)$ & $8(72.7)$ & 0.047 \\
Trimethoprim-sulphomethoxazole & 19 & $16(64.0)$ & $3(27.3)$ & $3(27.3)$ \\
Fusidic acid & $3(8.1)$ & $0(0)$ & $11(100)$ & 0.023 \\
Phosphomycin & $11(29.7)$ & $0(0)$ & & 0.001 \\
\hline
\end{tabular}

"n (\%): number/percentage

Table 2 shows the resistance patterns for MRSA and MRCoNS. A total of 12 resistance patterns were obtained. Differences were found between patterns obtained for MRSA and MRCoNS, with 7 different patterns for MRSA and 5 for MRCoNS.

\section{Discussion}

Our study showed that goats in the Canary Islands are frequently colonised by MRSA-ST398. The presence of these multiresistant bacteria had been previously studied in pigs and livestock workers on the island of Tenerife but they had not been isolated in goats (Morcillo et al., 2012). The presence of livestock-associated methicillin resistant Staphylococcus aureus (LA-MRSA) CC398 could indicate that these multiresistant strains have passed, in this habitat, from pigs to goats, due to some livestock contact in farms or even through human carriers. LA-MRSA CC398 strains have been described in many countries in Europe and worldwide as being prevalent in pigs and workers of the sector (Morcil- 
lo et al., 2012; Chuang and Huang, 2015; Sahibzada et al., 2017). Accordingly, various authors stressed the importance of the occupational hazard caused by LA-MRSA CC398, with a higher colonisation in exposed workers than in the overall population (Goerge et al., 2017; Anker et al., 2018).

Table 2

Resistance patterns of the strains studied

\begin{tabular}{lccc}
\hline \multirow{2}{*}{ Pattern } & Total (\%) & \multicolumn{2}{c}{ Microorganism } \\
\cline { 3 - 4 } & & MRSA (\%) & MRCoNS (\%) \\
\hline PG+OXA+STX & $10(27.8)$ & $10(40.0)$ & $0(0)$ \\
PG+OXA & $6(16.7)$ & $6(24)$ & $0(0)$ \\
PG+OXA+FM+E & $4(11.1)$ & $0(0)$ & $4(36.4)$ \\
PG+OXA+CC+E & $3(8.3)$ & $3(12.0)$ & $0(0)$ \\
PG+OXA+FM & $3(8.3)$ & $0(0)$ & $3(27.3)$ \\
PG+OXA+CC+STX & $2(5.6)$ & $2(8.0)$ & $0(0)$ \\
PG+OXA+CC+STX+FM+FA+E & $2(5.6)$ & $0(0)$ & $2(18.2)$ \\
PG+OXA+GM+TM+STX & $2(5.6)$ & $2(8.0)$ & $0(0)$ \\
PG+OXA+CC+STX+E+GM+TM & $1(2.8)$ & $1(4.0)$ & $0(0)$ \\
PG+OXA+CC+FM+E & $1(2.8)$ & $0(0)$ & $1(9.1)$ \\
PG+OXA+CC+FM+E+FA & $1(2.8)$ & $0(0)$ & $1(9.1)$ \\
PG+OXA+STX+E & $1(2.8)$ & $1(4.0)$ & $0(0)$ \\
& & &
\end{tabular}

*PG: benzylpenicillin, OXA: oxacillin, GM: gentamicin, TM: tobramycin, CC: clindamycin, E: erythromycin, SXT: trimethoprim-sulphamethoxazole, FA: fusidic acid, FM: phosphomycin

Several studies have reported the detection of MRSA strains in goat milk (Traversa et al., 2015; Parisi et al., 2016; Obaidat et al., 2018), considering it to be a major finding, as such strains had not been detected before in this type of livestock. Cortimiglia et al. (2015) demonstrated that the same MRSA strain was able to persist over time on the farm, being isolated from both the bulk tank milk and the udder of three goats. However, Porrero et al. (2012) did not find the me$c A$ gene to be present in $S$. aureus from goat and sheep mastitis samples in Spain.

There are very few studies on MRSA colonisation in healthy goats. Alves et al. (2009) indicated that, contrary to the high presence of MRSA in other animals such as pigs and horses, its prevalence in ruminants was very low. Aquino et al. (2012) analysed 23 nasal samples from goats and did not find methicillin-resistant staphylococci; however, they found them in the staff of a teaching and research farm. Loncaric et al. (2013) reported, for the first time in Austria, the infection of a goat by the MRSA ST 398 strain, as well as the colonisation of other goats from the same herd and its suspected transmission to humans, resulting in colonisation in a farmer in charge of feeding them. The study indicates that none of the animals or humans were on antibiotic treatment, nor did they show any clinical signs. 
We consider it important that our study has revealed the presence of strains with the mecA gene in different Staphylococcus species, as they are an important reservoir of resistance genes amounting to a serious public and animal health concern (Zhang et al., 2009; Huber et al., 2011; Nemeghaire et al., 2014).

The chromogenic medium used for the screening of MRSA $\left(\right.$ ChromID $^{\circledR}$ MRSA, (BioMérieux ${ }^{\circledR}$ SA) allowed us to detect the presence of other methicillinresistant coagulase-negative Staphylococcus bacteria. Other authors have used the same medium to detect MRCoNS strains (Nemeghaire et al., 2014). We thus consider that it could be an appropriate medium for screening methicillinresistant coagulase-negative staphylococci growing as white colonies.

Several studies show the presence of MRCoNS strains in various animals and animal-derived products, such as meat and milk (Bhargava and Zhang, 2014; Nemeghaire et al., 2014), and the importance of these strains in workers in contact with the animals (Huber et al., 2011). In our study, we have identified 6 different species colonising goat nostrils, S. lentus and S. sciuri being predominant. In a study carried out in the United States, Zhang et al. (2009) found the presence of MRCoNS strains in different farm animals, including 10 goats, identifying the same species that we did.

The strains in our study present high resistance to other non-beta-lactam antibiotics. MRSA strains were resistant, in a high percentage, to co-trimoxazole (75\%), minor in the case of MRCoNS, with significant differences. The main resistance pattern was multiresistance to non-carbapenem beta-lactams, together with co-trimoxazole which appears in $40 \%$ of the strains studied. Another important aspect to consider is resistance to erythromycin, clindamycin and to the aminoglycosides gentamicin and tobramycin.

MRCoNS strains present high resistance, especially to phosphomycin (100\% of the strains studied) and erythromycin $(72.7 \%)$, resistance to beta-lactams being the main pattern together with these two antimicrobials (36.4\%).

In 2014 Spain was the biggest user of veterinary antimicrobials among the countries that submitted data to the European Surveillance Project of Veterinary Antimicrobial Consumption and, according to the Spanish Agency of Medicines and Healthcare Products, sales continued to increase in 2015 (European Commission, 2016). We consider these resistance levels to be very high and, in our opinion, this could be related to the significant consumption of existing antibiotics in livestock in Spain.

It was a limitation of our study that we were authorised to perform only nasal screening. More studies, such as rectal colonisation screening, are needed for the surveillance of multidrug-resistant bacteria.

This study is the first to report MRSA ST398 and coagulase-negative Staphylococcus strains in goats in Spain, with a high resistance to also other groups of antibiotics. In conclusion, the high prevalence of MRSA and MRCoNS in the goats studied indicates the need to further study this strain of animal origin, as well as to increase surveillance and control measures at all stages of the food chain. 


\section{References}

Alves, P. D., McCulloch, J. A., Even, S., Le Maréchal, C., Thierry, A., Grosset, N., Azevedo, V., Rosa, C. A., Vautor, E. and Le Loir, Y. (2009): Molecular characterisation of Staphylococcus aureus strains isolated from small and large ruminants reveals a host rather than tissue specificity. Vet. Microbiol. 137, 190-195. doi: 10.1016/j.vetmic.2008.12.014

Anker, J. C. H., Koch, A., Ethelberg, S., Mølbak, K., Larsen, J. and Jepsen, M. R. (2018): Distance to pig farms as risk factor for community-onset livestock-associated MRSA CC398 infection in persons without known contact to pig farms. A nationwide study. Zoonoses Public Health 65, 352-360. doi: 10.1111/zph.12441

Aquino, V., Maluta, R. P. and de Ávila, F. A. (2012): Prevalence of methicillin-resistant staphylococci on a farm: staff can harbour MRS when animals do not. Zoonoses Public Health 59, 1-3. doi: 10.1111/j.1863-2378.2011.01413

Bhargava, K. and Zhang, Y. (2014): Characterization of methicillin-resistant coagulase-negative staphylococci (MRCoNS) in retail meat. Food Microbiol. 42, 56-60. doi: 10.1016/j.fm. 2014. 02.019

Chu, C., Yu, C., Lee, Y. and Su, Y. (2012): Genetically divergent methicillin-resistant Staphylococcus aureus and sec-dependent mastitis of dairy goats in Taiwan. BMC Vet. Res. 29, 39. doi: 10.1186/1746-6148-8-39

Chuang, Y. Y. and Huang, Y. C. (2015): Livestock-associated methicillin-resistant Staphylococcus aureus in Asia: an emerging issue? Int. J. Antimicrob. Agents 45, 334-340. doi: 10.1016/ j.ijantimicag.2014.12.007

Clinical Laboratory Standards Institute (2011): Performance Standards for Antimicrobial Susceptibility Testing; Twenty-First Informational Supplement Document M100-S21. CLSI, Wayne, PA. Available online: http://www.clsi.org/

Cortimiglia, C., Bianchini, V., Franco, A., Caprioli, A., Battisti, A., Colombo, L., Stradiotto, K., Vezzoli, F. and Luini, M. (2015): Short communication: Prevalence of Staphylococcus aureus and methicillin-resistant $S$. aureus in bulk tank milk from dairy goat farms in Northern Italy. J. Dairy Sci. 98, 2307-2311. doi: 10.3168/jds.2014-8923

Enright, M. C., Day, N. P. J., Davies, C. E., Peacock, S. J. and Spratt, B. G. (2000): Multilocus Sequence Typing for characterization of methicillin-resistant and methicillin-susceptible clones of Staphylococcus aureus. J. Clin. Microbiol. 38, 1008-1015.

European Commission (2016): Report 2016-8978. DG (SANTE) 2016-8887. Available in: http://ec.europa.eu/food/audits-analysis/overview reports

Feld, L., Bay, H., Angen, Ø., Larsen, A. R. and Madsen, A. M. (2018): Survival of LA-MRSA in dust from swine farms. Ann. Work. Expo. Health 62, 147-156. doi: 10.1093/annweh/wxx108

Fernandes Dos Santos, F., Mendonça, L. C., Reis, D. R. L., Guimarães, A. S., Lange, C. C., Ribeiro, J. B., Machado, M. A. and Brito, M. A. V. P. (2016): Presence of mecA-positive multidrug-resistant Staphylococcus epidermidis in bovine milk samples in Brazil. J. Dairy Sci. 99, 1374-1382. doi: 10.3168/jds.2015-9931

Goerge, T., Lorenz, M. B., van Alen, S., Hübne, N. O., Becker, K. and Köck, R. (2017): MRSA colonization and infection among persons with occupational livestock exposure in Europe: Prevalence, preventive options and evidence. Vet. Microbiol. 200, 6-12. doi: 10.1016/ j.vetmic.2015.10.027

Habrun, B., Račić, I., Beck, R., Budimir, A., Benić, M., Kompes, G., Špičić, S. and Cvetnić, Z. (2011): The presence of methicillin-resistant Staphylococcus aureus on large pig breeding farms in Croatia. Acta Vet. Hung. 59, 419-425. doi: 10.1556/AVet.2011.028

Hernández-Porto, M., Castro, B., Ramos, M. J., Arias, A., Aguirre-Jaime, A. and Lecuona, M. (2014): Risk factors for development of methicillin-resistant Staphylococcus aureuspositive clinical culture in nasal carriers after decolonization treatment. Am. J. Infec. Control 42, e75-79. doi: 10.1016/j.ajic.2014.03.011 
Huber, H., Ziegler, D., Pflüger, V., Vogel, G., Zweifel, C. and Stephan, R. (2011): Prevalence and characteristics of methicillin-resistant coagulase-negative staphylococci from livestock, chicken carcasses, bulk tank milk, minced meat, and contact persons. BMC Vet. Res. 7, 6. doi: 10.1186/1746-6148-7-6

Landeta, G., Curiel, J. A., Carrascosa, A. V., Muñoz, R. and de las Rivas, B. (2013): Characterization of coagulase-negative staphylococci isolated from Spanish dry cured meat products. Meat Sci. 93, 387-396. doi: 10.1016/j.meatsci.2012.09.019

Loncaric, I., Brunthaler, R. and Spergser, J. (2013): Suspected goat-to-human transmission of methicillin-resistant Staphylococcus aureus sequence type 398. J. Clin. Microbiol. 51, 1625-1626. doi: 10.1128/JCM.03052-12

Mahato, S., Mistry, H. U., Chakraborty, S., Sharma, P., Saravanan, R. and Bhandari, V. (2017): Identification of variable traits among the methicillin resistant and sensitive coagulase negative staphylococci in milk samples from mastitic cows in India. Front. Microbiol. 8, 1446. doi: 10.3389/fmicb.2017.01446.

Morcillo, A., Castro, B., Rodríguez-Álvarez, C., González, J. C., Sierra, A., Montesinos, M. I., Abreu, R. and Arias, Á. (2012): Prevalence and characteristics of methicillin-resistant Staphylococcus aureus in pigs and pig workers in Tenerife, Spain. Foodborne Pathog. Dis. 9, 207-210. doi: 10.1089/fpd.2011

Nemeghaire, S., Vanderhaeghen, W., Argudín, M. A., Haesebrouck, F. and Butaye, P. (2014): Characterization of methicillin-resistant Staphylococcus sciuri isolates from industrially raised pigs, cattle and broiler chickens. J. Antimicrob. Chemother. 69, 2928-2934. doi: $10.1093 / \mathrm{jac} / \mathrm{dku} 268$

Obaidat, M. M., Bani Salman, A. E. and Roess, A. A. (2018): High prevalence and antimicrobial resistance of mecA Staphylococcus aureus in dairy cattle, sheep, and goat bulk tank milk in Jordan. Trop. Anim. Health. Prod. 50, 405-412. doi: 10.1007/s11250-017-1449-7

Ortwine, J. K. and Bhavan, K. (2018): Morbidity, mortality, and management of methicillinresistant $S$. aureus bacteremia in the USA: update on antibacterial choices and understanding. Hospital Pract. (1995) 46, 64-72. doi: 10.1080/21548331.2018.1435128

Parisi, A., Caruso, M., Normanno, G., Latorre, L., Sottili, R., Miccolupo, A., Fraccalvieri, R. and Santagada, G. (2016): Prevalence, antimicrobial susceptibility and molecular typing of methicillin-resistant Staphylococcus aureus (MRSA) in bulk tank milk from southern Italy. Food Microbiol. 58, 36-42. doi: 10.1016/j.fm.2016.03.004

Pletinckx, L. J., Verhegghe, M., Crombé, F., Dewulf, J., De Bleecker, Y., Rasschaert, G., Butaye, P., Goddeeris, B. M. and De Man, I. (2013): Evidence of possible methicillin-resistant Staphylococcus aureus ST398 spread between pigs and other animals and people residing on the same farm. Prev. Vet. Med. 109, 293-303. doi: 10.1016/j.prevetmed.2012.10.019

Pomba, C., Rantala, M., Greko, C., Baptiste, K. E., Catry, B., van Duijkeren, E., Mateus, A., Moreno, M. A., Pyörälä, S., Ružauskas, M., Sanders, P., Teale, C., Threlfall, E. J., Kunsagi, Z., Torren-Edo, J., Jukes, H. and Törneke, K. (2017): Public health risk of antimicrobial resistance transfer from companion animals. J. Antimicrob. Chemother. 72, 957-968. doi: $10.1093 / \mathrm{jac} / \mathrm{dkw} 481$

Porrero, M. C., Hasman, H., Vela, A. I., Fernández-Garayzábal, J. F., Domínguez, L. and Aarestrup, F. M. (2012): Clonal diversity of Staphylococcus aureus originating from the small ruminants goats and sheep. Vet. Microbiol. 156, 157-161. doi: 10.1016/j.vetmic. 2011.10.015

Rodrigues, A. C., Belas, A., Marques, C., Cruz, L., Gama, L. T. and Pomba, C. (2018): Risk factors for nasal colonization by methicillin-resistant staphylococci in healthy humans in professional daily contact with companion animals in Portugal. Microbial Drug Res. 24, 434 446. doi: $10.1089 / \mathrm{mdr} .2017 .0063$

Sahibzada, S., Abraham, S., Coombs, G. W., Pang, S., Hernández-Jover, M., Jordan, D. and Heller, J. (2017): Transmission of highly virulent community-associated MRSA ST93 and live- 
stock-associated MRSA ST398 between humans and pigs in Australia. Scientific Reports 7, 5273. doi: 10.1038/s41598-017-04789-0

Sato, T., Usui, M., Maetani, S. and Tamura, Y. (2018): Prevalence of methicillin-resistant Staphylococcus aureus among veterinary staff in small animal hospitals in Sapporo, Japan, between 2008 and 2016: A follow up study. J. Infect. Chemother. 24, 588-591. doi: 10.1016/ j.jiac.2018.01.016

Shore, A. C. and Coleman, D. C. (2013): Staphylococcal cassette chromosome mec: recent advances and new insights. Int. J. Med. Microbiol. 303, 350-359. doi: 10.1016/j.ijmm. 2013.02.002

Smith, C. L., Klco, S. R. and Cantor, C. R. (1988): Pulsed-field gel electrophoresis and the technology of large DNA molecules. In: Davies, K. E. (ed.) Genome Analysis: A Practical Approach. Oxford IRL Press, Oxford, United Kingdom. pp. 41-72.

Sun, J., Yang, M., Sreevatsan, S., Bender, J. B., Singer, R. S., Knutson, T. P., Marthaler, D. G. and Davies, P. R. (2017): Longitudinal study of Staphylococcus aureus colonization and infection in a cohort of swine veterinarians in the United States. BMC Infec. Dis. 17, 690. doi: 10.1186/s12879-017-2802-1

Tenover, F. C., Arbeit, R. D., Goering, R. V., Mickelsen, P. A., Murray, B. E., Persing, D. H. and Swaminathan, B. (1995): Interpreting chromosomal DNA restriction patterns produced by pulsed-field gel electrophoresis: criteria for bacterial strain typing. J. Clin. Microbiol. 33, 2233-2239.

Traversa, A., Gariano, G. R., Gallina, S., Bianchi, D. M., Orusa, R., Domenis, L., Cavallerio, P., Fossati, L., Serra, R. and Decastelli, L. (2015): Methicillin resistance in Staphylococcus aureus strains isolated from food and wild animal carcasses in Italy. Food Microbiol. 52, 154-158. doi: 10.1016/j.fm.2015.07.012

Vanderhaeghen, W., Piepers, S., Leroy, F., Van Coillie, E., Haesebrouck, F. and De Vliegher, S. (2014): Effect, persistence, and virulence of coagulase-negative Staphylococcus species associated with ruminant udder health. J. Dairy Sci. 97, 5275-5293. doi: 10.3168/jds.20137775

Zhang, Y., Agidi, S. and LeJeune, J. T. (2009): Diversity of staphylococcal cassette chromosome in coagulase-negative staphylococci from animal sources. J. Appl. Microbiol. 107, 13751383. doi: $10.1111 /$ j. $1365-2672.2009 .04322 x$ 